\title{
Model Reduction of a Nonlinear Crash Model of a Racing Kart
}

Jörg Fehr, Dennis Grunert, Philip Holzwarth, Peter Eberhard

Institute of Engineering and Computational Mechanics (ITM)

\section{Motivation}

Automotive safety is a core area in the design process of new cars.

- hardware crash tests are expensive, simulation based design process $\Rightarrow$ many simulation runs are needed on HPC

nonlinear finite element equations

$$
\boldsymbol{M}_{\mathrm{e}} \cdot \ddot{\boldsymbol{q}}_{\mathrm{e}}+\boldsymbol{k}_{\mathrm{e}}\left(\boldsymbol{q}_{\mathrm{e}}, t\right)=\boldsymbol{h}_{\mathrm{e}}
$$

with mass matrix $\boldsymbol{M}_{\mathrm{e}} \in \mathbb{R}^{N \times N}$, elastic stiffness vector $\boldsymbol{k}_{\mathrm{e}}\left(\boldsymbol{q}_{\mathrm{e}}, t\right) \in \mathbb{R}^{N}$ and applied forces $\boldsymbol{h}_{\mathrm{e}} \in \mathbb{R}^{N}$ solved with explicit time solver (LS-DYNA)

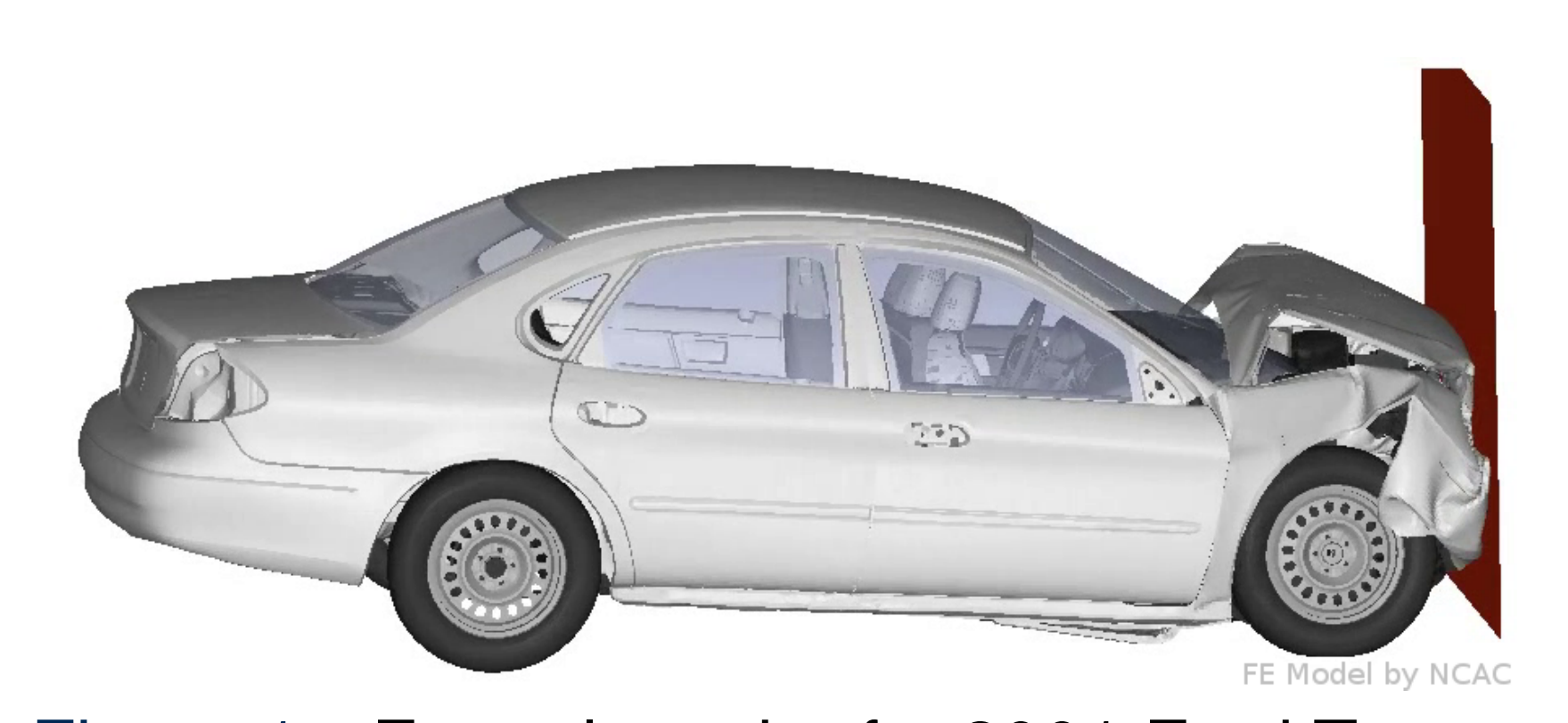

Figure 1: Frontal crash of a 2001 Ford Taurus.

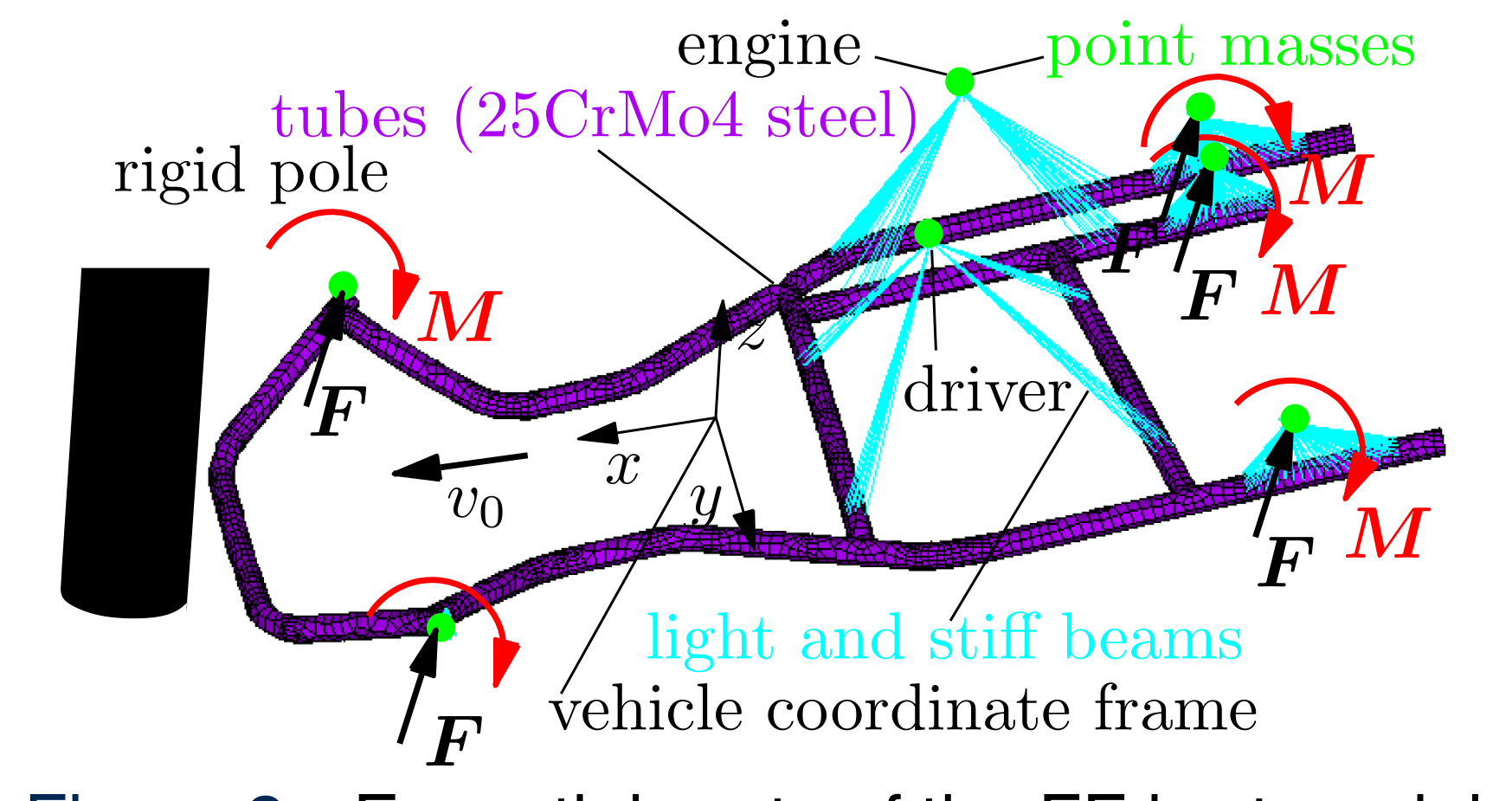

Figure 2: Essential parts of the FE kart model.

- tremendous simulation times and energy consumption due to highly detailed Finite Element models. Apply model order reduction for speedup

- for separation and understanding effects, a racing kart crashing against a pole as a surrogate model for research

- crash simulations possess three basic sources of nonlinearities in mechanics:

large deformation

L nonlinear material behavior

$\stackrel{\square}{\square}$ multiple contact scenarios

internal forces $\boldsymbol{k}_{\mathrm{e}}=\boldsymbol{k}_{\mathrm{e}}\left(\boldsymbol{q}_{\mathrm{e}}, \dot{\boldsymbol{q}}_{\mathrm{e}}, t, \ldots\right)$ are nonlinear

\section{Reduction Approach Substructuring:}

Separation of the model in parts with linear and parts with nonlinear behaviour [1]. Apply model reduction only to linear part with $\boldsymbol{k}_{\mathrm{e}}=\boldsymbol{K}_{\mathrm{e}} \cdot \boldsymbol{q}_{\mathrm{e}}$ ( $\boldsymbol{K}_{\mathrm{e}}$ is stiffness matrix). Linearization $\boldsymbol{K}_{\mathrm{e}} \approx \boldsymbol{K}_{\mathrm{e}}^{\text {tang }}=\partial \boldsymbol{k}_{\mathrm{e}} / \partial \boldsymbol{q}_{\mathrm{e}}$ around suitable working point using implicit solver option.

\section{Offline Step}

Identification of areas with small deformation (likely to behave linear): several offline simulations with varying initial velocity and position of the pole, see Figure 3.

- similar to passenger cars in frontal crashes, the plastic deformation in the rear of the kart is rather small

- separation of the model introducing $5 \times 15 \times 6=420$ interface dofs

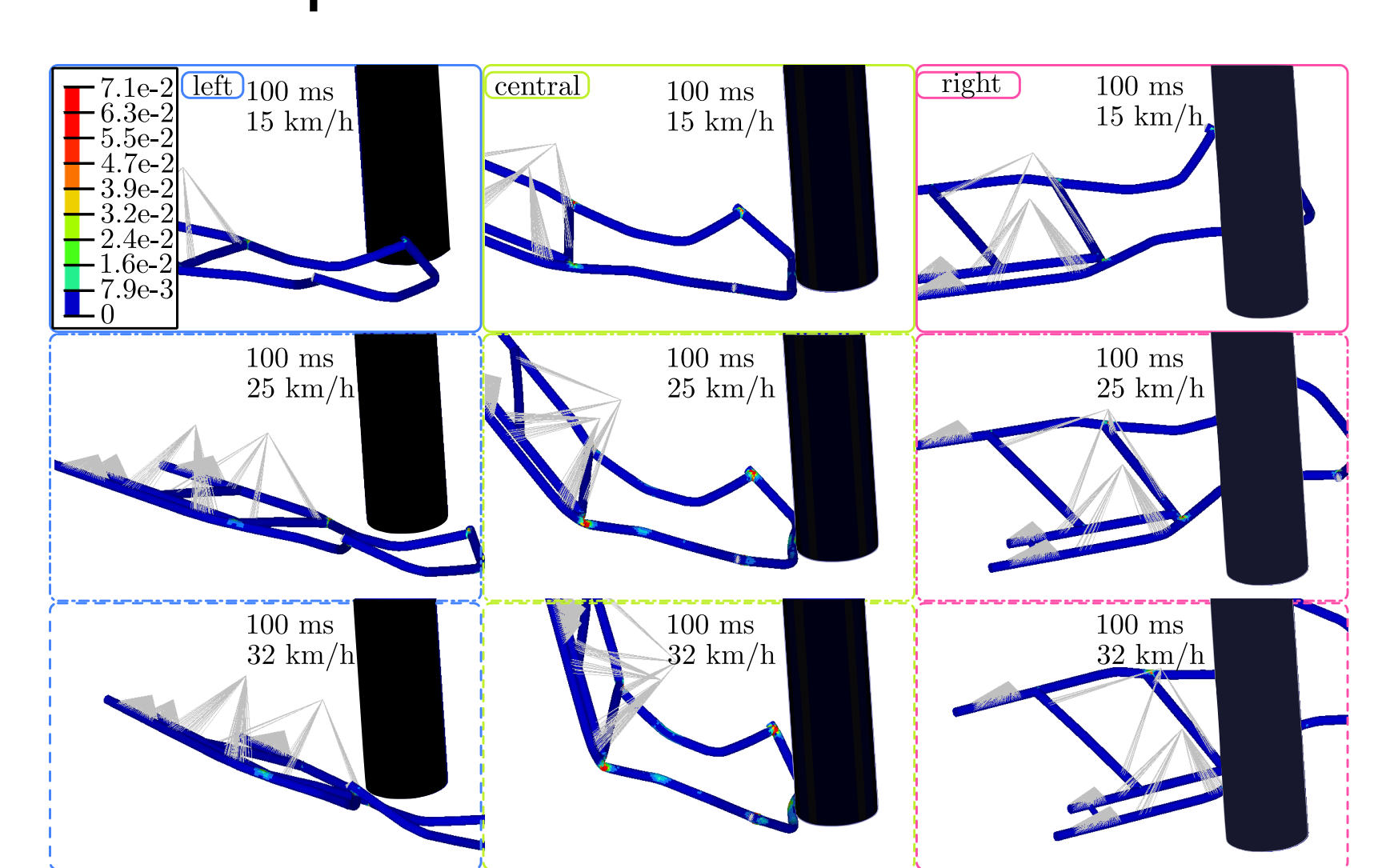

Figure 3: Nine offline simulations.

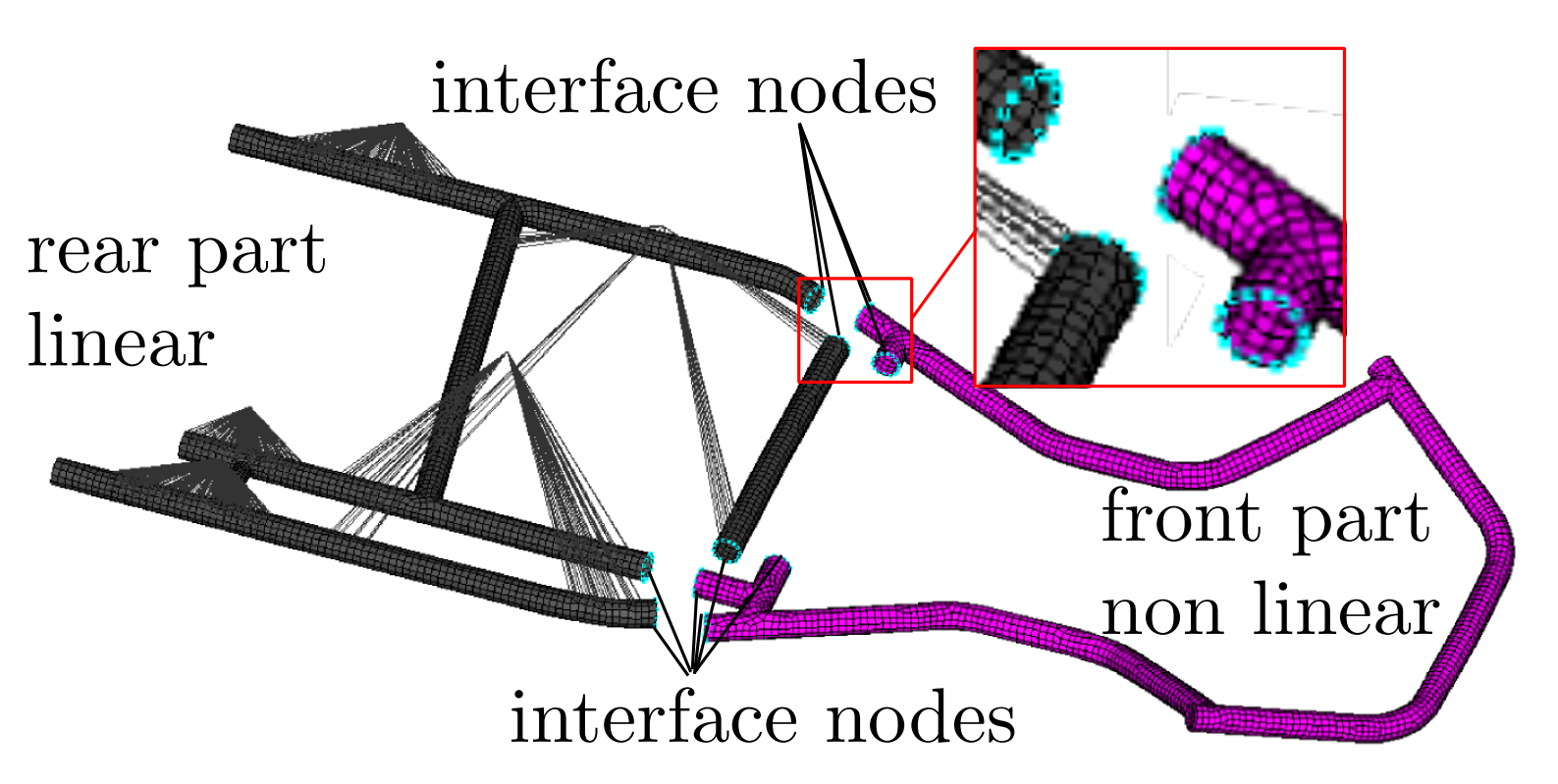

Figure 4: Separation in two parts and interface nodes at the rims.

\section{Interface Reduction}

static condensation:

$$
\left[\begin{array}{cc}
\boldsymbol{K}_{\mathrm{bb}} & \boldsymbol{K}_{\mathrm{bi}} \\
\boldsymbol{K}_{\mathrm{ib}} & \boldsymbol{K}_{\mathrm{ii}}
\end{array}\right] \cdot\left[\begin{array}{c}
\boldsymbol{I} \\
\boldsymbol{\Psi}
\end{array}\right]=\left[\begin{array}{l}
\boldsymbol{R} \\
\mathbf{0}
\end{array}\right]
$$

-420 interface modes

$\Rightarrow$ brute-force method interface approximation with $\Omega$ :

$$
\left[\begin{array}{ll}
\boldsymbol{K}_{\mathrm{bb}} & \boldsymbol{K}_{\mathrm{bi}} \\
\boldsymbol{K}_{\mathrm{ib}} & \boldsymbol{K}_{\mathrm{ii}}
\end{array}\right] \cdot\left[\begin{array}{l}
\Omega \\
\boldsymbol{\Psi}
\end{array}\right]=\left[\begin{array}{l}
\boldsymbol{R} \\
\mathbf{0}
\end{array}\right]
$$

- extract the most important deformation patterns at the interface, [4]

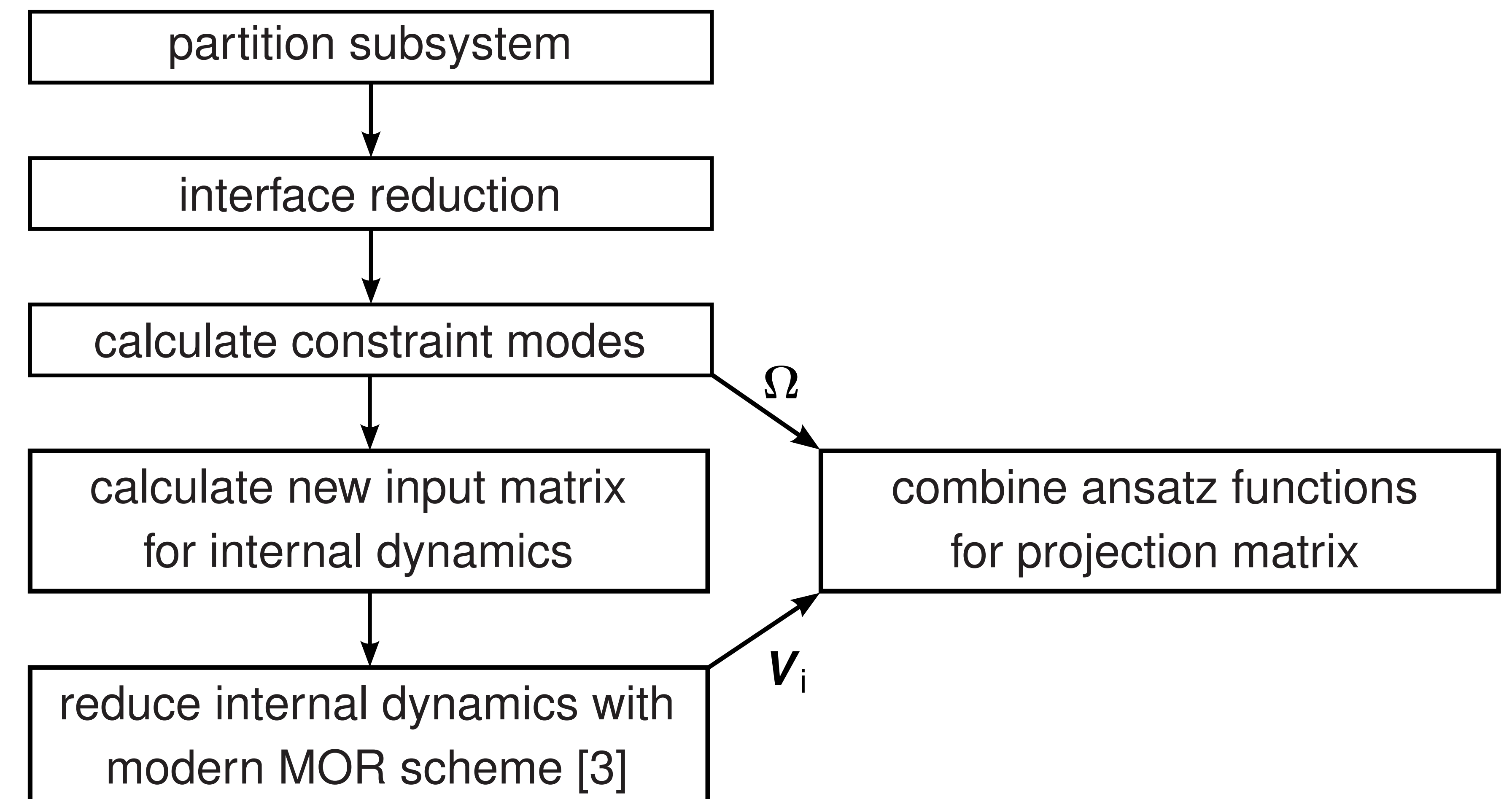
Figure 5: Overall reduction scheme: After partitioning of the system, the interface is reduced.
CMS-based reduction methods are prefered. Therefore, the constraint modes and the modes of the internal dynamics are calculated with advanced MOR.

\section{Optimal Reduction of Linear Substructures}

goal: find suitable methods for subsequent nonlinear simulation by linearized analysis

reduce both front and rear part $\Rightarrow$ separate basis reduction

$\left[\begin{array}{cc}\boldsymbol{M}_{1} & \mathbf{0} \\ \mathbf{0} & \boldsymbol{M}_{2}\end{array}\right]\left[\begin{array}{c}\ddot{\boldsymbol{q}}_{1} \\ \ddot{\boldsymbol{q}}_{2}\end{array}\right]+\left[\begin{array}{cc}\boldsymbol{K}_{1}+\boldsymbol{B}_{\mathrm{c}, 1} \boldsymbol{K}_{\mathrm{c}} \boldsymbol{B}_{\mathrm{c}, 1}^{\top} & -\boldsymbol{B}_{\mathrm{c}, 1} \boldsymbol{K}_{\mathrm{c}} \boldsymbol{B}_{\mathrm{c}, 2}^{\top} \\ -\boldsymbol{B}_{\mathrm{c}, 2} \boldsymbol{K}_{\mathrm{c}}^{\top} \boldsymbol{B}_{\mathrm{c}, 1}^{\top} & \boldsymbol{K}_{2}+\boldsymbol{B}_{\mathrm{c}, 2} \boldsymbol{K}_{\mathrm{c}} \boldsymbol{B}_{\mathrm{c}, 2}^{\top}\end{array}\right]\left[\begin{array}{l}\boldsymbol{q}_{1} \\ \boldsymbol{q}_{2}\end{array}\right]=\left[\begin{array}{l}\boldsymbol{B}_{\mathrm{i}, 1} \boldsymbol{u}_{1} \\ \boldsymbol{B}_{\mathrm{i}, 2} \boldsymbol{U}_{2}\end{array}\right]$

best interface reduction after analysis: global approach in combination with balanced truncation

\section{Nonlinear Simulation with Reduced Rear Part}

- Craig-Bampton: many interface nodes $\Rightarrow$ high eigenfrequency, therefore small maximum time step and high calculation time (over 11 days vs. 1 hour for unreduced model)

- good match until $30 \mathrm{~ms}$, phase shift after $30 \mathrm{~ms}$, see Figure 6, 7

v faster element processing ( 970 s vs. 2210 s) amortized by additional time to calculate reduced body (1260 s)

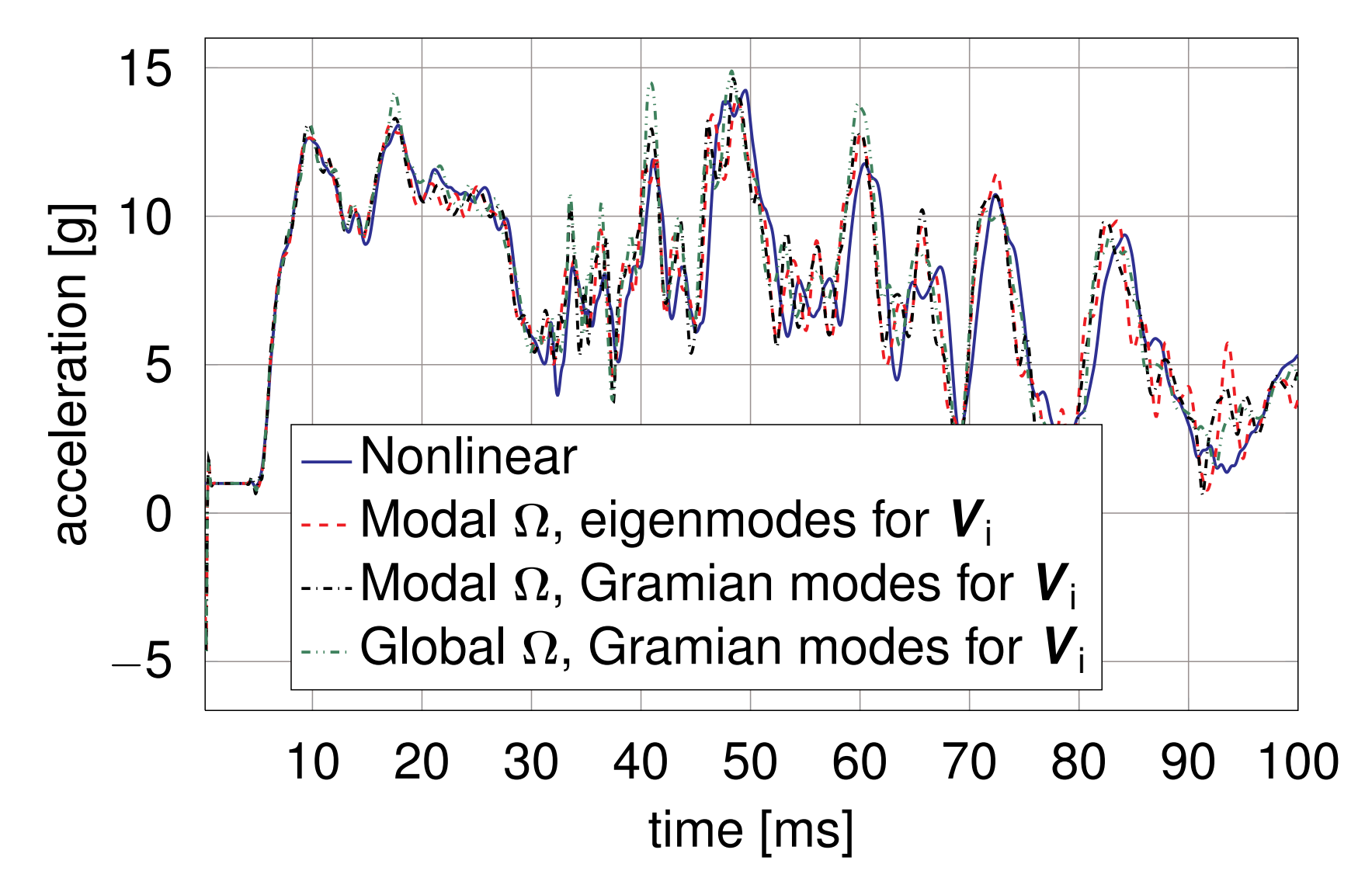

Figure 6: Acceleration of the driver calculated with different reduction approaches.

\section{Lessons Learned}

challenge: coupling between reduced and original model

- interface reduction is important

- orthogonalization with respect to rigid body modes
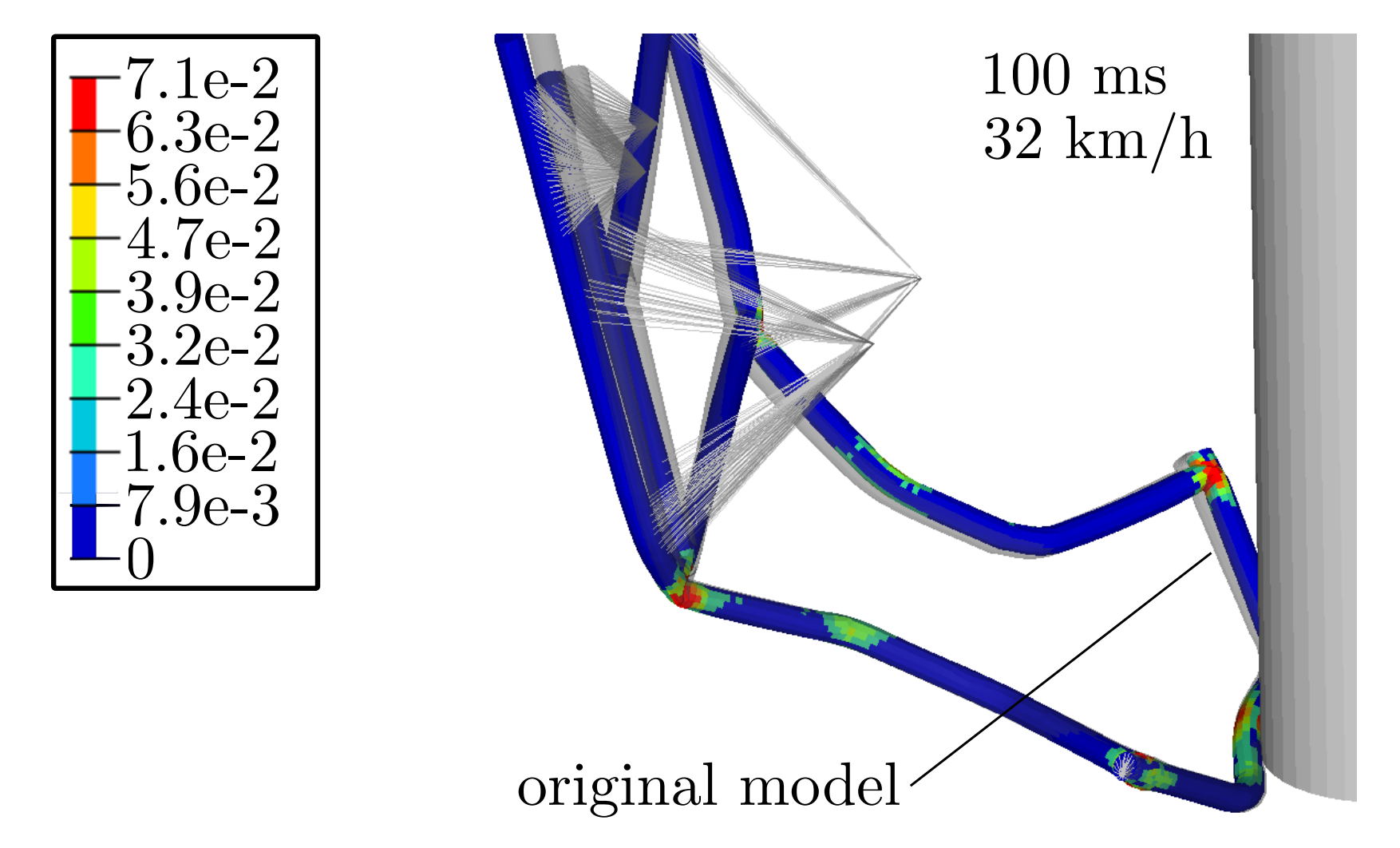

Figure 7: Plastic strain of the deformed, reduced model (CMS-Gram with modal interface ison.

\section{Future Steps}

- reduced simulation should be much faster by our experience

- apply method to full-scaled car nonlinear MOR for crash
[1] J. Fehr and D. Grunert.

Model reduction and clustering techniques for crash simulations. Proceedings in Applied Mathematics and Mechanics, 2015. or publication.

[2] J. Fehr, P. Holzwarth, and P. Eberhard.

Interface and model reduction for efficient simulations of nonlinear vehicle crash models. submitted to Mathematical and Computer Modelling of Dynamical Systems.

[3] P. Holzwarth and P. Eberhard.

SVD-based improvements for component mode synthesis in elastic multibody systems. European Journal of Mechanics - A/Solids, 49:408-418, 2015.

[4] Philip Holzwarth and Peter Eberhard.

Interface reduction for CMS methods and alternative model order reduction. In Proceedings of the MATHMOD 2015 - 8th Vienna International Conference on Mathematical Modelling, Vienna, Austria, 2015. 